archives-ouvertes

\title{
Distorted own-body representations in patients with dizziness and during caloric vestibular stimulation
}

Christophe Lopez

\section{To cite this version:}

Christophe Lopez. Distorted own-body representations in patients with dizziness and during caloric vestibular stimulation. Journal of Neurology, Springer Verlag, 2018, 265 (S1), pp.86-94. 10.1007/s00415-018-8906-8 . hal-02121665

\section{HAL Id: hal-02121665 https://hal-amu.archives-ouvertes.fr/hal-02121665}

Submitted on 6 May 2019

HAL is a multi-disciplinary open access archive for the deposit and dissemination of scientific research documents, whether they are published or not. The documents may come from teaching and research institutions in France or abroad, or from public or private research centers.
L'archive ouverte pluridisciplinaire HAL, est destinée au dépôt et à la diffusion de documents scientifiques de niveau recherche, publiés ou non, émanant des établissements d'enseignement et de recherche français ou étrangers, des laboratoires publics ou privés. 


\title{
Distorted own-body representations in patients with dizziness and during caloric vestibular stimulation
}

\author{
Christophe Lopez $^{1,5} \cdot$ Estelle Nakul $^{1} \cdot$ Nora Preuss $^{2} \cdot$ Maya Elzière $^{3} \cdot$ Fred W. Mast $^{4}$
}

\begin{abstract}
There is increasing evidence that vestibular disorders evoke deficits reaching far beyond imbalance, oscillopsia and spatial cognition. Yet, how vestibular disorders affect own-body representations, in particular the perceived body shape and size, has been overlooked. Here, we explored vestibular contributions to own-body representations using two approaches. Study 1 measured the occurrence and severity of distorted own-body representations in 60 patients with dizziness and 60 healthy controls using six items from the Cambridge Depersonalization Scale. $12 \%$ of the patients have experienced distorted own-body representations (their hands or feet felt larger or smaller), 37\% reported abnormal sense of agency, $35 \%$ reported disownership for the body, and $22 \%$ reported disembodiment. These proportions were larger in patients than controls. Study 2 aimed at testing whether artificial stimulation of the vestibular apparatus produced comparable distortions of own-body representations in healthy volunteers. We compared the effects of right-warm/leftcold caloric vestibular stimulation (CVS), left-warm/right-cold CVS and sham CVS on internal models of the left and right hands using a pointing task. The perceived length of the dorsum of the hand was increased specifically during left-warm/ right-cold CVS, and this effect was found for both hands. Our studies show a vestibular contribution to own-body representations and should help understand the complex symptomatology of patients with dizziness.
\end{abstract}

Keywords Vestibular disorder $\cdot$ Body schema $\cdot$ Depersonalization $\cdot$ Derealization $\cdot$ Out-of-body experience $\cdot$ Bodily selfconsciousness

This manuscript is part of a supplement sponsored by the German Federal Ministry of Education and Research within the funding initiative for integrated research and treatment centers.

\section{Christophe Lopez}

christophe.lopez@univ-amu.fr

1 Aix Marseille Univ, CNRS, LNSC, Marseille, France

2 Department of Neuroscience, Karolinska Institutet, Stockholm, Sweden

3 Centre des Vertiges, Hôpital Européen, Marseille, France

4 Department of Psychology, University of Bern, Bern, Switzerland

5 Laboratoire de Neurosciences Sensorielles et Cognitives-UMR 7260, Aix Marseille Univ and Centre National de la Recherche Scientifique (CNRS), Centre Saint-Charles, Fédération de Recherche 3C-Case B, 3, Place Victor Hugo, 13331 Marseille Cedex 03, France

\section{Introduction}

Research in neuroscience has highlighted the role of vestibular signals in functions that reach far beyond the control of eye movements and posture. To cite only a few of them, vestibular signals have been involved in spatial cognition and memory, affective control, mood, decision-making, perspective taking, awareness, and bodily self-consciousness (for recent reviews see [1, 2]). This apparent omnipotence of the vestibular system implies that damage to the vestibular receptors and vestibulo-thalamo-cortical pathways may evoke a large spectrum of perceptual and cognitive disorders.

Indeed, how vestibular disorders affect own-body representations, in particular, the perceived body shape and size, have been widely overlooked, despite anecdotal reports since the nineteenth century (reviewed in [3]). Bonnier [4] and Schilder [5], for example, collected cases of patients whose body felt enlarged or smaller, whose self appeared strange or unreal, or who felt disembodied during vertigo crises. These 
seminal descriptions paved the way to the study of depersonalization-derealization (DD) symptoms in otoneurological patients. DD combines the experience that the self appears strange or unreal, as if one is an outside observer of oneself, with the sensation that the environment is strange or unreal. Studies found that DD is more frequent and more severe in patients with dizziness than in healthy controls [6-8]. Yet, to date, we are lacking measurements of the occurrence and severity of distorted own-body representations in patients with dizziness.

In our previous studies, we proposed that distorted ownbody and self representations may result from a perceptual incoherence due to a failure to coherently integrate vestibular signals with somatosensory and visual signals about one's own body configuration, orientation and movement $[3,8]$. This proposition is supported by neuroimaging studies showing that several areas involved in own-body and self representations-such as the temporo-parietal junction, insula, intraparietal sulcus, premotor and cingulate cortexintegrate vestibular signals with somatosensory and visual signals [9]. These areas could be the neural correlate of these perceptual conflicts.

Studies have endeavoured to test this hypothesis by interfering with the neural bases of own-body representations using artificial vestibular stimulation in neurologically normal individuals. These studies yielded results that partially diverge. While some authors found that caloric vestibular stimulation (CVS) and galvanic vestibular stimulation (GVS) modified the perceived size of the hands and thighs [10, 11], others found no effect [12]. Similarly, different results have been found regarding the effects of CVS and GVS on ownership for real and fake body parts $[12,13]$. In addition to this, the comparison of left vs. right CVS and GVS in tasks measuring body representations produced equivocal results [12-14].

Here, we explored vestibular contributions to own-body representations using two approaches. Study 1 measured the occurrence of distorted own-body representation in otoneurological patients. Study 2 tested whether artificial vestibular stimulation could similarly distort own-body representations in healthy volunteers. As there is a right hemispheric dominance for vestibular information processing [15], we compared the effects of left vs. right CVS on internal models of the left and right hand.

\section{Materials and methods}

\section{Study 1: distorted bodily representations in patients with dizziness}

\section{Participants}

In this observational study, we included 60 patients suffering from dizziness and vertigo (40 females; mean age \pm SD $50.9 \pm 14.6$ years): 47 patients had peripheral vestibular disorders including benign paroxysmal positional vertigo (BPPV), Meniere's disease, perilymphatic fistula, or other acute unilateral vestibular disorders, as the most common aetiology. Two patients had central forms of dizziness, one had somatoform dizziness, and one had dizziness of another origin. The origin of dizziness was unknown in nine patients. Depending on the symptoms, otoneurological examination included videonystagmographic examination of spontaneous, positional and head-shaking test nystagmus, pendular rotatory test and caloric test, video head impulse test (VHIT), cervical vestibular-evoked myogenic potentials (cVEMPs), and MRI. Patients were compared to 60 age- and gender-matched controls with no history of otoneurological and psychiatric disorders. Table 1 summarizes the socio-demographic characteristics of the two populations. The institutional Ethics Committee (Hôpital Européen, CEDP-HE16.03) approved all procedures.

Patients and controls were matched for age, gender, education level, employment status and alcohol consumption, whereas they differed with respect to their marital status and smoking habits (Table 1). At the time of the examination, $80 \%$ of the patients presented with a spontaneous nystagmus $(n=14)$, positional nystagmus $(n=34)$ and/or head-shaking nystagmus $(n=32)$. Objective sign of vestibular dysfunction was evidenced by decreased gain of the horizontal vestibulo-ocular reflex (VHIT gain $<0.8$ ), decreased caloric vestibular response (deficit $>25 \%$ ), and absent or asymmetrical cVEMPs, in $87 \%$ of the patients who received these tests.

\section{Data recording and analysis}

The same otoneurologist recruited all patients and controls, and the same procedures were explained to both groups. Patients filled out the Cambridge Depersonalization Scale [16], which includes 29 items introduced as follows: This questionnaire describes strange and 'funny' experiences that normal people may have in their daily life. We are interested in their: (a) frequency, i.e., how often have you had these experiences [since you had dizziness for the first time (patients)/over the last 6 months 
Table 1 Demographic and clinical characteristics of patients with dizziness and healthy controls

\begin{tabular}{|c|c|c|c|}
\hline & $\begin{array}{l}\text { Patients with } \\
\text { dizziness } \\
(n=60)\end{array}$ & $\begin{array}{l}\text { Healthy } \\
\text { controls } \\
(n=60)\end{array}$ & $\begin{array}{l}\text { Patients vs. con- } \\
\text { trols }\end{array}$ \\
\hline Age (years $\pm S D)$ & $50.9 \pm 14.6$ & $50.8 \pm 14.5$ & $t=-0.04 ; P=1.00$ \\
\hline Females/males & $40 / 20$ & $40 / 20$ & - \\
\hline \multicolumn{4}{|c|}{ Highest education level (\%) } \\
\hline Level 1 & 40 & 33 & \multirow[t]{5}{*}{$\chi^{2}=2.26 ; P=0.68$} \\
\hline Level 2 & 20 & 25 & \\
\hline Level 3 & 14 & 18 & \\
\hline Level 4 & 14 & 8 & \\
\hline Level 5 & 11 & 15 & \\
\hline \multicolumn{4}{|c|}{ Employment status (\%) } \\
\hline Employed & 60 & 69 & \multirow[t]{4}{*}{$\chi^{2}=1.61 ; P=0.66$} \\
\hline Student & 2 & 2 & \\
\hline Retired & 23 & 20 & \\
\hline Unemployed & 15 & 8 & \\
\hline \multicolumn{4}{|l|}{ Marital status (\%) } \\
\hline Single & 14 & 7 & \multirow[t]{3}{*}{$\chi^{2}=8.76 ; P=0.01$} \\
\hline Married/couple & 54 & 80 & \\
\hline $\begin{array}{l}\text { Divorced/wid- } \\
\text { owed }\end{array}$ & 32 & 13 & \\
\hline Smokers (\%) & 32 & 13 & $\chi^{2}=5.90 ; P=0.01$ \\
\hline \multicolumn{4}{|c|}{ Alcohol consumption (\%) } \\
\hline No alcohol & 63 & 49 & \multirow[t]{3}{*}{$\chi^{2}=3.04 ; P=0.22$} \\
\hline $1-5$ units/week & 27 & 42 & \\
\hline$>5$ units/week & 10 & 8 & \\
\hline $\begin{array}{l}\text { History of } \\
\text { dizziness } \\
\quad(\text { months } \pm \mathrm{SD})\end{array}$ & $46.2 \pm 76.4$ & - & - \\
\hline Migraine (\%) & 31 & 17 & $\chi^{2}=3.07 ; P=0.08$ \\
\hline
\end{tabular}

Education level according to the French education system; level 1: before high school; level 2: accomplished high school; level 3: two years after high school; level 4: bachelor's degree, level 5: master's degree, engineering degree, $\mathrm{PhD}, \mathrm{MD}$

(controls)]; and (b) their approximate duration. For each item, participants indicated the frequency of the experience on a scale ranging from 0 ("never") to 4 ("all the time"), and its duration on a scale from 1 ("few seconds") to 6 ("more than a week"). For each item, the global score (sum of the frequency and duration) ranged from 0 to 10. Although we have presented global analyses elsewhere [8], here we analyse items measuring distortions of the body schema, body ownership, agency and disembodiment (Q3, Q12, Q20, Q23, Q24 and Q27; Fig. 1). Multivariate analyses of variance (MANOVAs) were calculated (IBM SPSS Chicago, IL, USA) to clarify how several aspects of the vestibular pathology influence DD. Scores for Q3, Q12, Q20, Q23, Q24 and Q27 were the dependent variables, and the presence of a nystagmus, positive VHIT, positive caloric test, abnormal VEMPs and migraine, were fixed factors.

\section{Study 2: measurement of hand representation in healthy participants}

\section{Participants}

The data were obtained from 16 healthy, right-handed participants ( 9 females; mean age \pm SD $23.8 \pm 6.6$ years). The Ethics Committee of the Faculty of Human Sciences, University of Bern, approved experimental procedures. All participants gave written informed consent.

\section{Caloric vestibular stimulation}

We used CVS with a constant air flow injected into the auditory canals through a short plastic tube (Airmatic II, GN Otometrics, Taastrup, Denmark; see detailed procedures in [10]). Warm air $\left(47^{\circ} \mathrm{C}\right)$ was injected in one ear and at the same time cold air $\left(20^{\circ} \mathrm{C}\right)$ was injected in the opposite ear. Bilateral CVS as opposed to unilateral CVS has the advantage that it better corresponds to the physiological responses during real head rotations, and it is thus a less conflicting stimulation. To control for unspecific (attentional, tactile and auditory) effects of CVS, we applied a sham CVS-a constant air flow at body temperature $\left(37^{\circ} \mathrm{C}\right)$ simultaneously in both ears. This produces identical test conditions, but with no vestibular stimulation.

After the experiment, we monitored eye movements during $45 \mathrm{~s}$ of CVS and sham CVS by videonystagmography (eVNG, BioMed, Jena, Germany) [10]. The nystagmus was not recorded, but the experimenter controlled that CVS evoked a nystagmic response. We included in the final analysis only those participants who exhibited a clear nystagmus during CVS. No nystagmus was evoked by sham CVS.

\section{Localization task}

Participants were blindfolded and their left or right hand was positioned palm down under a digitizing tablet on a table in front of them, so that their hand was aligned with their mid-sagittal body plane (adapted from [10, 17]). Participants were asked to locate five anatomical landmarks on the dorsal surface of their left or right hand: the knuckle of the little finger, the knuckle of the middle finger, the knuckle of the index finger, the tip of the middle finger and the wrist. Each landmark was initially shown to the participant. Participants indicated the location of the landmarks using a stylus and the digitizing tablet recorded the $x$ - and $y$-coordinates the participants were pointing to.

The localization of anatomical landmarks was conducted during one session of left-warm/right-cold CVS, 
A

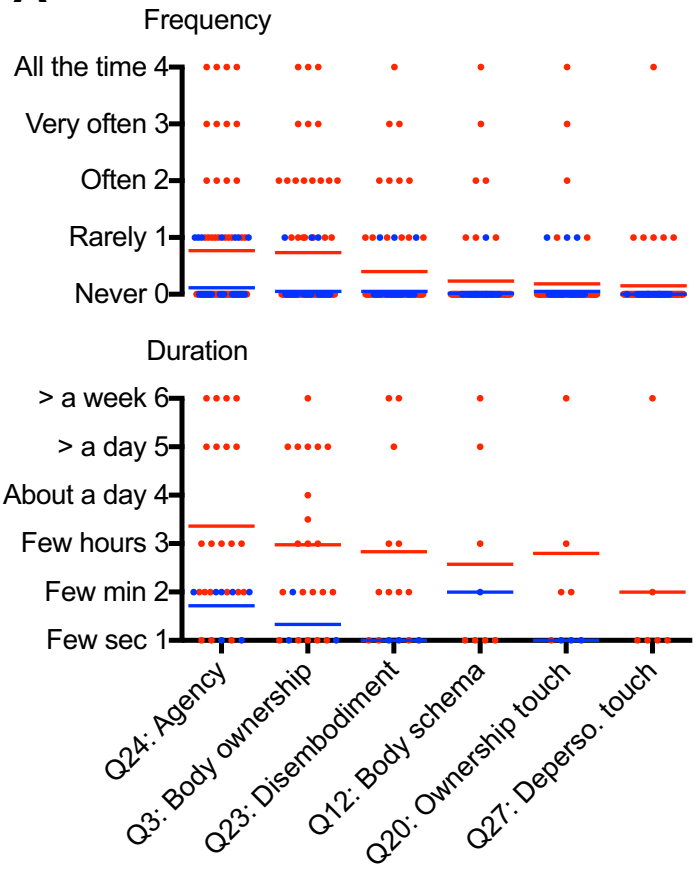

B

Total score

(frequency + duration)



C

$\%$ of individuals

with symptoms

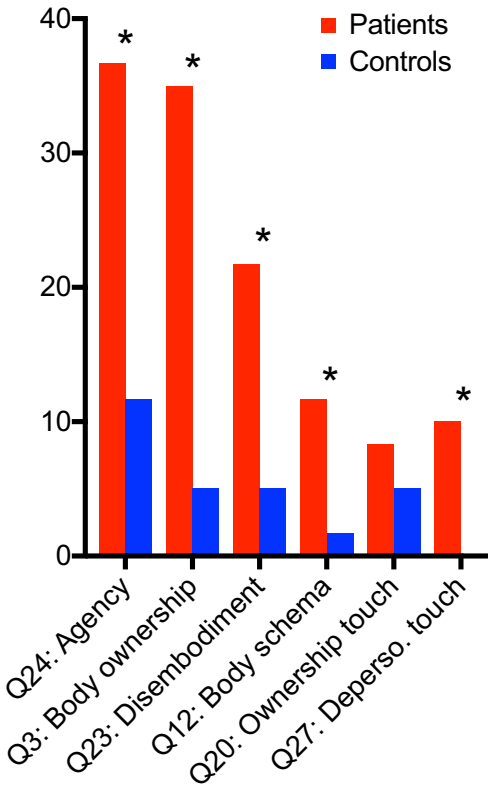

Fig. 1 Own-body perception in patients with dizziness. a Dots represent individual ratings of frequency and duration for each of the six items pertaining to the sense of the body (red dots: patients; blue dots: controls). Q24: "When I move it doesn't feel as if I were in charge of the movements, so that I feel 'automatic' and mechanical as if I were a "robot"; Q3: "Parts of my body feel as if they didn't belong to me"; Q23: "I have the feeling of being outside my body"; Q12: "I have the feeling that my hands or my feet have become larger or smaller"; Q20: "I cannot feel properly the objects that I touch with

one session of right-warm/left-cold CVS and one session of sham CVS, for both the left and right hand, presented in a counterbalanced order across participants. Participants performed the pointing task when lying backwards in a reclined position and while receiving CVS. For each CVS condition and each hand, participants judged the location of the five anatomical landmarks in separate blocks. Each block consisted of pointing consecutively ten times to the same anatomical landmark. The order in which they had to locate the landmarks was randomized across participants.

For each participant, we calculated the mean $x$ - and $y$-coordinates of the perceived position of each landmark. We calculated the perceived length of the dorsum of the hand (Euclidean distance between the knuckle of the middle finger and the wrist), the width of the hand (Euclidean distance between the knuckle of the little finger and the knuckle of the index finger) and the length of the middle finger (Euclidean distance between the tip and knuckle of the middle finger). After each CVS condition, participants were turned back to the upright position for $5 \mathrm{~min}$ of rest. my hands for it feels as if it were not me who were touching it"; Q27: "I have to touch myself to make sure that I have a body or a real existence". Red and blue horizontal lines indicate the mean. b Histograms show the average score (sum of frequency and duration) for the six questionnaire items ordered by their perceived intensity. Error bars represent the standard error of mean. $* P<0.05$, Mann-Whitney test. c Histograms represent the percentage of individuals that answered positively to each item questionnaire. ${ }^{*} P<0.05, z$ test for proportion

\section{Subjective reports}

Participants filled out a questionnaire designed to evaluate vertigo/illusory self-motion and side effects after each session of CVS and sham stimulation (from [13, 14]). They reported the magnitude of their sensations on a 7-point scale ranging from 0 ("absent") to 6 ("severe"). The effectiveness of CVS was furthermore assessed by the same participants filling-out the Cox and Swinson DD questionnaire [18] after each session of CVS and sham CVS [10]. This questionnaire was chosen as it is shorter than the Cambridge Depersonalization Scale [16]. Participants rated the intensity of 28 items using a 5-point scale ranging from 0 ("does not occur") to 4 ("very severe"). 


\section{Results}

\section{Study 1: distorted bodily representations in patients with dizziness}

As shown in Fig. 1a, patients with dizziness responded positively more frequently to questionnaire items related to bodily disorders than the healthy controls. In particular, they experienced bodily disorders more frequently, and for a longer duration ("more than a week" for some patients), than did healthy controls (all of them experienced DD never or rarely, for a few seconds to a few minutes only). Figure $1 \mathrm{~b}, \mathrm{c}$ shows that only few patients (12\%) explicitly reported experiencing distortions of their body schema (i.e., the feeling that their hands or feet have become larger or smaller; Q12). Yet, the proportion of patients reporting this was significantly higher than in controls (two samples $z$ test for proportion: $z=2.84, P=0.004$ ). In addition, intensity of this sensation was significantly stronger in patients than controls (Mann-Whitney test, $U=1620$, $z=-2.19, P=0.041)$. About one-third of the patients reported abnormal agency (i.e., the feeling of not being in charge of their actions, Q24). More patients experienced abnormal agency $(z=4.64, P<0.0001)$ with stronger intensity than controls $(U=1301, z=-3.49, P<0.0001)$. Importantly, more patients reported abnormal body ownership (Q3) than healthy controls $(z=5.81, P<0.0001)$, and the intensity of body "disownership" was significantly higher in patients than controls $(U=1239.5, z=-4.21$, $P<0.0001)$. Feelings of "disembodiment" were more pronounced in patients compared to controls $(\mathrm{Q} 23 ; z=3.72$, $P=0.0002)$ and with stronger intensity $(U=1483.5$, $z=-2.81, P=0.002)$. Moreover, more patients experienced touch as depersonalized (Q27; $z=3.30, P=0.0001$ ) with stronger intensity $(U=1620, z=-2.50, P=0.027)$ compared to controls. Ownership for touch (Q20) had similar intensity between patients and controls. In conclusion, our results indicate that vestibular disorders precipitate distortions of the body schema, abnormal sense of agency and of body ownership, as well as sensations of being disembodied. This is surprising since questions about bodily disorders are not part of the clinical routine, and the patients we tested were not preselected on the basis of previous reports. Further research is necessary to better investigate whether distortions of the body schema occur systematically under particular clinical and perhaps personality-based preconditions.

Using Pillai's trace in the MANOVAs, there was no significant effect of the nystagmus on DD scores $(V=0.10$, $\left.F_{5,52}=1.0, P=0.44\right)$, suggesting no influence of oscillopsia. In addition, there was no significant effect of migraine $\left(V=0.17, F_{6,48}=1.63, P=0.16\right)$, abnormal VHIT
$\left(V=0.27, F_{6,26}=1.59, P=0.19\right)$, VEMPs $\left(V=0.13, F_{6,20}\right.$ $=0.49, P=0.81)$, and caloric test $\left(V=0.15, F_{6,23}=0.69\right.$, $P=0.66)$ on DD.

\section{Study 2: measurement of hand representation in healthy participants}

Figure 2a depicts the internal model of the hand inferred from the pointing tasks. We analysed separately the perceived length of the dorsum of the hand, hand width and finger length using repeated-measures ANOVAs with vestibular stimulation (sham CVS, right-warm/left-cold CVS, leftwarm/right-cold CVS) and hand (left, right) as within-subject factors. The ANOVA revealed a significant main effect of vestibular stimulation only for the length of the dorsum of the hand $\left[F_{2,30}=6.97, P=0.003, \eta_{p}{ }^{2}=0.32\right]$ (Fig. 2b). The perceived length of the hand was significantly increased during left-warm/right-cold CVS when compared to sham CVS $(P=0.0017$, two-sided paired $t$ test $)$ and to right-warm/ left-cold CVS $(P=0.04)$. There was also a significant effect of Hand $\left[F_{1,15} \mathrm{v} 12.06, P=0.003, \eta_{p}{ }^{2}=0.45\right]$, with larger left than right hand perception. Despite similar patters of numerically larger hand dimension for left-warm/right-cold CVS, there was no significant effect of vestibular stimulation for the hand width $\left[F_{2,30}=1.16, P=0.33, \eta_{p}{ }^{2}=0.07\right]$ and the finger length $\left[F_{2,30}=0.43, P=0.65, \eta_{p}{ }^{2}=0.03\right]$. There was no other significant main effect or interaction.

Questionnaire data analysis revealed that the type of CVS modulated the intensity of vertigo/illusory self-motion (Friedman's ANOVA, $P=0.003$ ) (Fig. 2c). The intensity of vertigo was significantly stronger during right-warm/leftcold CVS (Wilcoxon signed-rank test: $Z=2.75, P=0.006$ ) and left-warm/right-cold CVS $(Z=3.03, P=0.002)$ when compared to sham CVS. Vertigo was similar during right and left CVS $(Z=0.70, P=0.48)$, indicating that the reported difference between left and right CVS on hand size perception cannot be accounted for by differences in the intensity of CVS-evoked vertigo. Interestingly, there was no significant effect of the type of CVS on general discomfort (Friedman's ANOVA, $P=0.096)$ and nausea $(P=0.341)$, ruling out unspecific effects of CVS on the perceived hand size (Fig. 2c). The analysis of the DD questionnaire revealed an overall main effect of the type of CVS $(P=0.001)$ (Fig. 2d). DD scores were significantly higher during right-warm/leftcold CVS $(Z=2.68, P=0.007)$ and left-warm/right-cold CVS $(Z=3.17, P=0.002)$ when compared to sham CVS. Total scores of DD were similar during right and left CVS $(Z=0.60, P=0.55)$. Finally, we found no significant correlation between the distortions of hand size perception during right-warm/left-cold CVS or left-warm/right-cold CVS and the intensity of illusory self-motion, as well as, no correlation with DD scores. 
A



B

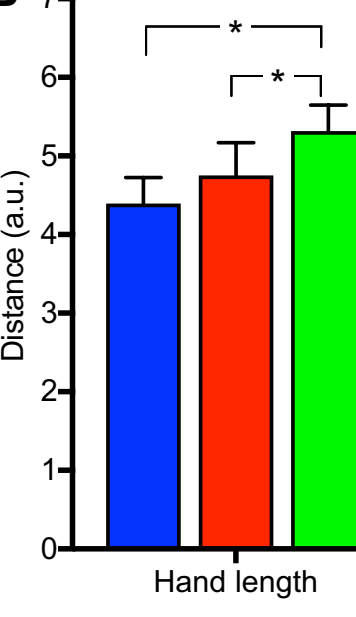

C

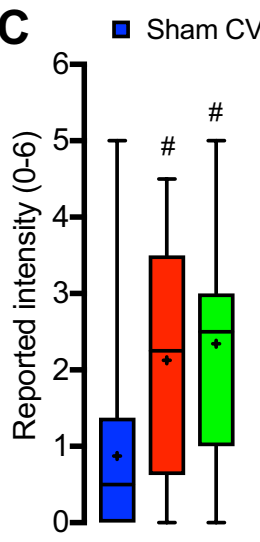

Vertigo

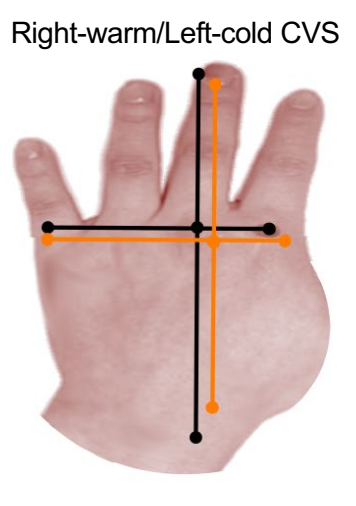

Left-warm/Right-cold CVS

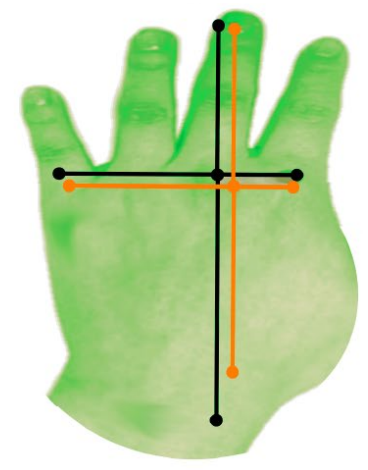

Sham CVS

口 Right-warm/Left-cold CVS

口 Left-warm/Right-cold CVS

\section{Discussion}

\section{Study 1}

We assessed distortions of own-body representations in patients with dizziness: $12 \%$ of the patients experienced their hands or feet have become larger or smaller. This is, to our knowledge, the first attempt to quantify the occurrence of perceived own-body distortions in vestibular patients. Other questionnaires have assessed the "strangeness" or "numbness" of the body [6], but we specifically asked for metric properties of body parts (Q12) [16]. We found cases of patients with dizziness whose body distortions extended to the neck, face, or entire body $[4,5,8,19]$. Altered size of perceived body parts is reminiscent of asomatognosia (macrosomatognosia/hyperschématie; microsomatognosia/ 
4Fig. 2 Influence of caloric vestibular stimulation on the internal model of the body. a Schematic depiction of the perceived hand size calculated after participants pointed repeatedly to the tip and knuckle of their middle finger, the knuckle of their little finger, the knuckle of their index finger, and their wrist. The average Euclidean distance between these five anatomical landmarks is represented by black lines for the left hand and orange lines for the right hand on a picture of a left hand. For all types of CVS the perceived hand representation was characterized by shortened middle fingers and broadened dorsum of the hand. Left-warm/right-warm CVS increased the perceived size of both hands. b The mean perceived length of the dorsum of the hand, hand width and middle finger length is represented for each type of CVS. Although a main effect of vestibular stimulation was found only for the length of the hand, it is represented for the hand width and finger length for illustration purpose. Error bars represent the standard error of mean. ${ }^{*} P<0.05$, two-sided paired $t$ test. c Box-and-Whisker plots illustrate the intensity $(0=$ absent; $6=$ severe $)$ of vertigo/illusory self-motion, general discomfort and nausea reported after each block of stimulation. The top and bottom ends of the whisker represent the 95th and 5th percentiles of the distribution, the horizontal line inside the box represents the median, the black cross represents the mean. "Differences with respect to sham CVS $(P<0.05$, two-sided Wilcoxon signed-ranks test). d Total score of DD measured with the Cox and Swinson's questionnaire [18] after each block of stimulation. Same conventions as for part $\mathrm{C}$

hyposchématie [4]) encountered in neurological disorders, such as Alice in Wonderland syndrome, migraine, stroke and epilepsy. Distortions of the body schema are also commonly observed during limb anaesthesia, with illusions of swelling, elongation or shortening of the limbs [20]. Interestingly, distortions of own-body representations do not only arise from central lesions or somatosensory deficits but also from vestibular disorders.

Our results indicate that distortions of own-body representations were less frequent in vestibular disorders than abnormal agency, body ownership and disembodiment. More than one-third of the patients experienced abnormal agency and body ownership, confirming older case reports and group analyses indicating that vertigo can distort bodily self-consciousness [4-7, 21]. Agency and ownership are both deemed crucial to establish a minimal sense of self, as the self is considered the agent of the action (' $I$ ' have moved or 'I' have been moved) and ownership over the body provides a sense of 'mineness'. We note that there has been to date no direct, objective, measure of the vestibular contribution to agency. By contrast, there is evidence in healthy participants that GVS influences illusory ownership over a fake hand $[12,13]$, and this again suggests a tight connection between vestibular input and hand representation. In addition, our data show that over $20 \%$ of the patients reported the feeling of being outside of their body. A recent prospective study in 210 patients with dizziness showed that $14 \%$ of them reported having had an out-of-body experience, a proportion significantly higher than in healthy controls [8]. As vestibular sensations are frequently associated with out-ofbody experiences [8], and because vestibular stimulation in healthy controls modulate the anchoring of the visuospatial perspective to the body [22], vestibular signals should play a central role in anchoring the self to the body.

To account for distorted own-body representations, agency, body ownership and disembodiment in our patients, we propose that vestibular disorders create multisensory conflicts and perceptual incoherence, reshaping bodily experiences $[3,10]$. This proposition is supported by a large body of data indicating that multisensory conflicts-especially between visual and somatosensory signals - can evoke mislocalization of the self and ownership over another body in healthy participants (reviewed in [9]). Another potential cause of distorted own-body representation is migraine. Despite a trend for higher proportion of migraine in our patients with dizziness than in controls (as reported previously [23]), we found no relation between migraine and the severity of DD. Future investigations should compare DD in patients with vestibular migraine and other forms of migraine. From a clinical perspective, these findings are relevant and demonstrate that-unlike current practices in conventional clinical routines-signs of distorted body representations can be an important source of information, for diagnostics and rehabilitation alike. The underlying mechanisms, however, can be more rigorously investigated by means of experimental paradigms in healthy participants. The purpose of the second study was to test whether artificial stimulation of the vestibular apparatus produced comparable distortions of own-body representations in healthy participants.

\section{Study 2}

We show that left-warm/right-cold CVS increased the perceived length of the dorsum of the hand. These data confirm that vestibular signals are important for constructing a coherent model of the body. Along this line, Schönherr and May [11] showed that cold CVS decreased the estimated thigh width, but did not show a difference between left and right CVS. Our data indicate distortions restricted to the length of the dorsum of the hand, and no effect on the hand width and length of the finger. Similarly, Ferrè et al. [24] showed that during GVS touches applied to the dorsum of the hand, but not to the fingers, were mislocalized towards the wrist, i.e., along the proximal-distal axis. Similar to our study, which found no effect on hand width, these authors [24] found no effect of GVS for the ulnar-radial axis.

Another important finding of our present study was the dominant effect of left-warm/right-cold CVS on the body schema. This finding is coherent with a dominant effect of left-cathodal GVS reported for the localization of touch on the hand [24], mislocalization of the hand during the rubber hand illusion [12], and mental own-body imagery [14]. Other studies have reported opposite results, with a 
dominance of right-cathodal GVS for illusory ownership over a fake hand [13] and embodiment [22], or found no dominance of left vs. right vestibular stimulation. Thus, more studies are needed to understand whether there is a functional asymmetry of the vestibular system for own-body representations, and whether this asymmetry is task specific. If we consider that a common set of brain areas processes vestibular information and underpins own-body representations, our results may suggest a more extensive overlap of areas activated by left-warm/right-cold CVS with areas involved in own-body representations. For example, activation of the parietal operculum and insula was more bilateral after left than right vestibular nerve stimulation [25]. Thus, left vestibular nerve stimulation is likely to interfere more with bilateral structures involved in own-body representations, including the secondary somatosensory cortex, temporo-parietal junction and posterior insula [26]. In the same vein, there is evidence that left vestibular deafferentation is more likely to impair the egocentric reference frame [27]. Finally, we found no interaction between the type of CVS and the hand tested. This lack of predictable directional effects may be related to the fact that CVS interacts with internal models of the body harbored in bilateral secondary somatosensory areas, and temporo-parietal junction, where somatosensory receptive fields are large and bilateral. Functional neuroimaging revealed ipsilateral pathways from the peripheral somatosensory system and, interestingly, colocalization of touch and vestibular processing in the bilateral secondary somatosensory cortex $[28,29]$.

\section{Limitations}

A first limitation of study 1 is that it relies on self-report assessment. It can be difficult for patients to verbalize the phenomenology of their dizziness symptoms. A second limitation is the heterogeneity of the aetiologies included. This is due to the prospective nature of our study, reflecting the recruitment of patients with dizziness in a specialized ENT-otoneurological centre. The sample of our study does not allow to compare the effects of different categories of vestibular disorders on own-body representations. Yet, our data allow to conclude that, overall, patients with dizziness are more prone to distorted own-body representations. In addition, our data indicate that clinical findings, such as the presence of nystagmus, migraine or impaired semicircular canal functions, do not predict the severity of DD. Future prospective studies should investigate in large samples of patients whether acute vs. chronic vestibular disorders, unilateral vs. bilateral vestibular disorders, or whether disorders characterized by a vestibular loss $v s$. a paroxysmal vestibular irritation, are more likely to precipitate DD.

\section{Conclusions}

Both studies show a vestibular contribution to internal models of the body. We believe that the present work helps understand the complex symptomatology of patients with dizziness, who present with deficits reaching far beyond the well-known imbalance and oscillopsia, including alterations of the most fundamental aspects of the self.

Acknowledgements The research leading to these results has received funding from the People Programme (Marie Curie Actions) of the European Union's Seventh Framework Programme (FP7/2007-2013) under REA Grant agreement number 333607 ('BODILYSELF, vestibular and multisensory investigations of bodily self-consciousness'), and the SINERGIA Grant "Balancing Self and Body" of the Swiss National Science Foundation (125135).

\section{Compliance with ethical standards}

Conflicts of interest The authors declare they have no conflict of interest.

Ethical standard Ethical approvals have been obtained from the local ethics committees (Study 1: Hôpital Européen, Marseille CEDPHE 16.03; Study 2: Committee of the Faculty of Human Sciences, University of Bern) and participants provided informed consent in accordance with the Declaration of Helsinki.

\section{References}

1. Lopez C (2016) The vestibular system: balancing more than just the body. Curr Opin Neurol 29:74-83. https://doi.org/10.1097/ WCO.0000000000000286

2. Mast FW, Preuss N, Hartmann M, Grabherr L (2014) Spatial cognition, body representation and affective processes: the role of vestibular information beyond ocular reflexes and control of posture. Front Integr Neurosci 8:44. https://doi.org/10.3389/ fnint.2014.00044

3. Lopez C (2013) A neuroscientific account of how vestibular disorders impair bodily self-consciousness. Front Integr Neurosci 7:91. https://doi.org/10.3389/fnint.2013.00091

4. Bonnier P (1905) L'Aschématie. Rev Neurol Paris 12:605-609

5. Schilder P (1935) The image and appearance of the human body. International Universities Press, New York

6. Sang FY, Jauregui-Renaud K, Green DA et al (2006) Depersonalisation/derealisation symptoms in vestibular disease. J Neurol Neurosurg Psychiatry 77:760-766

7. Tschan R, Wiltink J, Adler J et al (2013) Depersonalization experiences are strongly associated with dizziness and vertigo symptoms leading to increased health care consumption in the German general population. J Nerv Ment Dis 201:629-635. https://doi.org/10.1097/NMD.0b013e3182982995

8. Lopez C, Elzière M (2017) Out-of-body experience in vestibular disorders-a prospective study of 210 patients with dizziness. Cortex. https://doi.org/10.1016/j.cortex.2017.05.026

9. Blanke O (2012) Multisensory brain mechanisms of bodily self-consciousness. Nat Rev Neurosci 13:556-571. https://doi. org/10.1038/nrn3292 
10. Lopez C, Schreyer HM, Preuss N, Mast FW (2012) Vestibular stimulation modifies the body schema. Neuropsychologia 50:1830-1837. https://doi.org/10.1016/j.neuropsychologi a.2012.04.008

11. Schönherr A, May CA (2016) Influence of caloric vestibular stimulation on body experience in healthy humans. Front Integr Neurosci 10:14. https://doi.org/10.3389/fnint.2016.00014

12. Ferrè ER, Berlot E, Haggard $P$ (2015) Vestibular contributions to a right-hemisphere network for bodily awareness: combining galvanic vestibular stimulation and the "Rubber Hand Illusion". Neuropsychologia 69C:140-147. https://doi.org/10.1016/j.neuro psychologia.2015.01.032

13. Lopez C, Lenggenhager B, Blanke O (2010) How vestibular stimulation interacts with illusory hand ownership. Conscious Cogn 19:33-47. https://doi.org/10.1016/j.concog.2009.12.003 pii]

14. Lenggenhager B, Lopez C, Blanke O (2008) Influence of galvanic vestibular stimulation on egocentric and object-based mental transformations. Exp Brain Res 184:211-221

15. Dieterich M, Brandt $T$ (2018) Global orientation in space and the lateralization of brain functions. Curr Opin Neurol 31:96-104. https://doi.org/10.1097/WCO.0000000000000516

16. Sierra M, Berrios GE (2000) The Cambridge Depersonalization Scale: a new instrument for the measurement of depersonalization. Psychiatry Res 93:153-164

17. Longo MR, Haggard P (2010) An implicit body representation underlying human position sense. Proc Natl Acad Sci USA 107:11727-11732. https://doi.org/10.1073/pnas.1003483107

18. Cox BJ, Swinson RP (2002) Instrument to assess depersonalization-derealization in panic disorder. Depress Anxiety 15:172-175

19. Rode G, Vallar G, Revol P et al (2012) Facial macrosomatognosia and pain in a case of Wallenberg's syndrome: selective effects of vestibular and transcutaneous stimulations. Neuropsychologia 50:245-253. https://doi.org/10.1016/j.neuropsychologi a.2011.11.018
20. Paqueron X, Leguen M, Rosenthal D et al (2003) The phenomenology of body image distortions induced by regional anaesthesia. Brain J Neurol 126:702-712

21. Grigsby JP, Johnston CL (1989) Depersonalization, vertigo and Meniere's disease. Psychol Rep 64:527-534

22. Pavlidou A, Ferrè ER, Lopez C (2018) Vestibular stimulation makes people more egocentric. Cortex doi. https://doi. org/10.1016/j.cortex.2017.12.005

23. Lempert T, Neuhauser H (2009) Epidemiology of vertigo, migraine and vestibular migraine. J Neurol 256:333-338. https:// doi.org/10.1007/s00415-009-0149-2

24. Ferrè ER, Vagnoni E, Haggard P (2013) Vestibular contributions to bodily awareness. Neuropsychologia 51:1445-1452. https://doi. org/10.1016/j.neuropsychologia.2013.04.006

25. Fink GR, Marshall JC, Weiss PH et al (2003) Performing allocentric visuospatial judgments with induced distortion of the egocentric reference frame: an fMRI study with clinical implications. Neuroimage 20:1505-1517

26. Corradi-Dell'Acqua C, Tomasino B, Fink GR (2009) What is the position of an arm relative to the body? Neural correlates of body schema and body structural description. J Neurosci 29:41624171. https://doi.org/10.1523/JNEUROSCI.4861-08.2009

27. Saj A, Honoré J, Bernard-Demanze L et al (2013) Where is straight ahead to a patient with unilateral vestibular loss? Cortex 49:1219-1228. https://doi.org/10.1016/j.cortex.2012.05.019

28. Kanno A, Nakasato N, Hatanaka K, Yoshimoto T (2003) Ipsilateral area $3 \mathrm{~b}$ responses to median nerve somatosensory stimulation. Neuroimage 18:169-177

29. Bottini G, Paulesu E, Gandola M et al (2005) Left caloric vestibular stimulation ameliorates right hemianesthesia. Neurology 65:1278-1283 